\title{
Multi-Kilowatt Peak Power Nanosecond Er-Doped Fiber Laser
}

\author{
V. V. Dvoyrin, D. Klimentov, J. O. Klepsvik, I. V. Mazaeva, and I. T. Sorokina
}

\begin{abstract}
We report a two-stage diode-pumped Er-doped fiber amplifier operating at the wavelength of $1550 \mathrm{~nm}$ at the repetition rate of $10-100 \mathrm{kHz}$ with an average output power of up to $10 \mathrm{~W}$. The first stage comprising Er-doped fiber was corepumped at the wavelength of $1480 \mathrm{~nm}$, whereas the second stage comprising double-clad Er/Yb-doped fiber was clad-pumped at the wavelength of $975 \mathrm{~nm}$. The estimated peak power for the 0.4-nm full-width at half-maximum laser emission at the wavelength of $1550 \mathrm{~nm}$ exceeded 4-kW level. The initial 100-ns seed diode laser pulse was compressed to $3.5 \mathrm{~ns}$ as a result of the 34-dB total amplification. The observed 30-fold efficient pulse compression reveals a promising new nonlinear optical technique for the generation of high power short pulses for applications in eye-safe ranging and micromachining.
\end{abstract}

Index Terms-Fiber lasers, infrared lasers, optical fibers.

\section{INTRODUCTION}

A CTIVELY Q-switched nanosecond fiber lasers, particularly those operating in the eye-safe spectral domain in the vicinity of $1.55 \mu \mathrm{m}$ are highly demanded for numerous applications, i.e., material processing, medicine, sensing [1], and ranging [2], [3]. The emission of Er-doped fiber laser $(1.55-1.61 \mu \mathrm{m})$ fits well the transparency window of the atmosphere. It makes Er-doped fiber laser an excellent candidate for the light detection and ranging applications (LIDARs) [4]-[10], even in the bad weather conditions (rainy, foggy or snowy). This is essentially important for the ranging on the sea, where the weather is rather changeable and sometimes unpredictable.

Laser peak power and pulse duration are responsible for coverage and resolution of a LIDAR system, whereas pulse repetition rate defines the data acquisition rate. Modern inexpensive semiconductor detectors can resolve pulses of duration of several nanoseconds, and laser systems with high repetition rates of $10-100 \mathrm{kHz}$ allow observation in real-time. Amplified distributed feedback (DFB) laser diodes producing short pulses with a narrow linewidth are widely used in traditional LIDAR systems. Such lasers have low average and peak powers at milliwatt level that leads to difficulties in development of a high-power laser system. Recently, the peak power of $7.6 \mathrm{~kW}$ was reported for the Er/Yb-doped master-oscillatorpower-amplifier (MOPA) laser system producing 1.2-ns pulses at a repetition rate of $50 \mathrm{kHz}$ with pulse energy of $9.6 \mu \mathrm{J}$ [3]. The pulse energy of $290 \mu \mathrm{J}$ was achieved in the Er/Yb-doped fiber amplifier chain [4]. This system had a moderate peak power $(2 \mathrm{~kW})$ and relatively long pulses of 100-ns duration. Large mode area fiber amplifiers allow producing higher pulse peak powers and energies [11]-[15]. However, this type of laser sources require the use of free-space optical components, including hybrid bulk-fiber configuration, leading to the poor pulse control and the more stringent requirements to the pump diodes. Other promising approaches towards obtaining pulsed laser action in fiber lasers, that have been implemented, include, e.g. all-fiber oscillators [16], [17], hybrid fiber systems with waveguide saturable absorbers [18], etc. Whereas such techniques are under development now, for practical applications the most straightforward and promising concept is the MOPA configuration with a seed laser diode.

In this letter, we report the development of the Er-doped all-fiber MOPA operating at the transparency window of the atmosphere and generating eye-safe 3.5-ns pulses. The MOPA was based on the FBG-stabilized seed laser diode as opposed to DFB laser diodes normally used in Yb- or Er-doped fiber amplifiers. High output average and peak power of the seed diode laser as compared to typically implemented DFB laser diodes allowed obtaining the peak power of more than $4 \mathrm{~kW}$. However, the most intriguing observation in the course of the work was that the initially relatively long pulses of $100-\mathrm{ns}$ were drastically compressed down to only few nanoseconds as a result of the amplification revealing a useful nonlinear optical technique for short pulse generation.

\section{EXPERIMENTAL}

The fiber-coupled FBG-stabilized semiconductor laser diode (Princeton Lightwave Inc.) operating at the wavelength of $1550 \mathrm{~nm}$ was used as a seed laser for the fiber amplifier, which consisted of the two amplification stages (Fig. 1). The first amplification stage consisted of a piece of the Er-doped silicabased fiber with core diameter of $4 \mu \mathrm{m}$. It was core-pumped with two fiber-coupled laser diodes (Fitel Inc.) operating at 


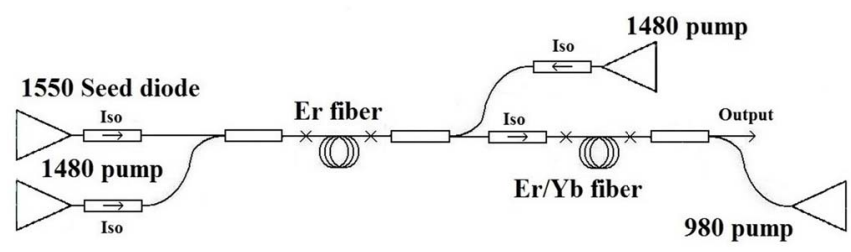

Fig. 1. The schematic of the setup.

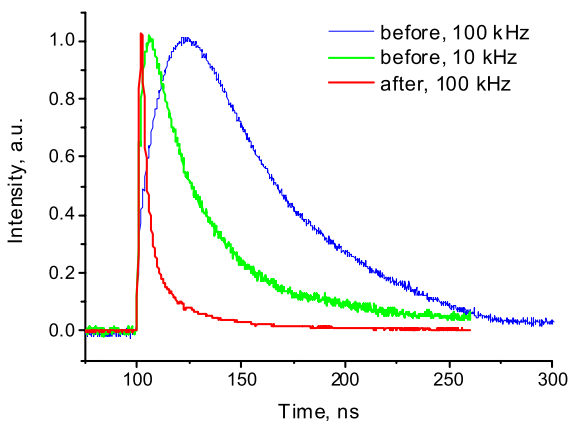

Fig. 2. Pulse shapes before and after the $2^{\text {nd }}$ stage amplifier.

the wavelength of $1480 \mathrm{~nm}$ with up to $300 \mathrm{~mW}$ optical power each, $600 \mathrm{~mW}$ in total. The second amplification stage utilized a 3-m long piece of a double-clad $\mathrm{Yb} /$ Er-doped silicabased fiber with core and clad diameters of 12 and $130 \mu \mathrm{m}$, respectively. It was clad-pumped at the wavelength of $975 \mathrm{~nm}$ by a fiber-coupled laser diode (IPG Photonics Inc.) in the counter-propagation direction with up to $40 \mathrm{~W}$ optical power. The piece of passive output fiber that was spliced to the active fiber was a single mode fiber with $8^{\circ}$ angle-cleaved facet preventing the back reflection. The output radiation was a lowest order mode providing an excellent nearly Gaussian beam quality [19]. Pulse trains were recorded with an oscilloscope of $0.5 \mathrm{GHz}$ band and a fast InGaAs semiconductor detector with $\sim 1 \mathrm{~ns}$ response time. The emission spectra were measured with an optical spectrum analyzer; a thermal power meter was used for the measurements of the output optical power.

\section{RESULTS}

It was observed that the laser produced a stable pulse train in the whole range of the repetition rates from 10 to $100 \mathrm{kHz}$ at the pump power of about $5 \mathrm{~W}$. The laser output emission was studied at the rates of 10 and $100 \mathrm{kHz}$. At the maximum current, the seed laser diode produced $\sim 100$-ns pulses with the repetition rate of $100 \mathrm{kHz}$ and the average optical power of $4 \mathrm{~mW}$. The average optical power emitted from the first amplification stage amounted to $360 \mathrm{~mW}$ corresponding to $20 \mathrm{~dB}$ optical gain. The energy extraction from the amplifier was equal to $60 \%$. A weak pulse compression down to $65 \mathrm{~ns}$ was already detected (Fig. 2). The full width at half maximum (FWHM) of the laser line amounted to $\sim 0.4 \mathrm{~nm}$ and remained nearly unchanged in all the experiments.

After the propagation through the second-stage amplifier, the pulse duration drastically dropped down. It experienced nearly exponential fall with the pump power increase (Fig. 3). The pulse was compressed down to $3.5 \mathrm{~ns}$ at $9.5 \mathrm{~W}$ of the output power. At $38 \mathrm{~W}$ of the pump power the average output
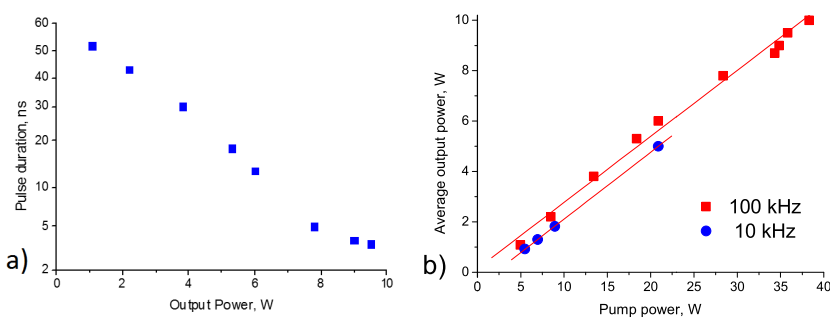

Fig. 3. Pulse compression (a) and laser efficiency (b) in the $2^{\text {nd }}$ stage amplifier.

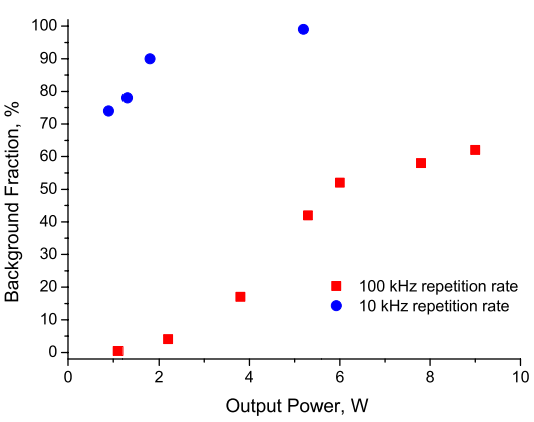

Fig. 4. Fraction of the spectral background in the total output radiation.

power amounted to $10 \mathrm{~W}$; the corresponding amplifier efficiency was equal to $26 \%$ ( $40 \%$ quantum efficiency). No change in the spectral width of the laser line was detected. However, we observed a rise of a broad spectral background, which was negligible at low output powers. Thus, at $1 \mathrm{~W}$ of the output power it corresponded to less than $0.5 \%$ from the total output power. On the contrary, at the output power of $9 \mathrm{~W}$ more than a half of the laser radiation must be attributed to the background (Fig. 4). It is worth noting that the spectral background in the spectra of the emission from the $1^{\text {st }}$-stage amplifier was negligible.

At the moment, we attribute this background to the amplified spontaneous emission (ASE) known to be the usual reason for similar phenomena in the high-power amplifiers with high optical gain. The estimation of the peak power of the emission under the narrow 1550-nm line (excluding the background fraction from the total output power) is about $4 \mathrm{~kW}$ for the most intensive 3.5-ns pulse.

The optical power of the seed diode operating at $10 \mathrm{kHz}$ repetition rate was equal to $0.6 \mathrm{~mW}$. The optical power of $160 \mathrm{~mW}$ corresponding to the optical gain of $24 \mathrm{~dB}$ was obtained after the first amplification stage. At the repetition rate of $10 \mathrm{kHz}$ the initial pulse was compressed down to $25 \mathrm{~ns}$ (Fig. 2).

The slope efficiency of the $2^{\text {nd }}$ stage amplifier operating at the repetition rate of $10 \mathrm{kHz}$ was similar to the previous case consisting of $26 \%$. At the output power from the $2^{\text {nd }}$ stage amplifier of $0.9 \mathrm{~W}$, the pulse duration already amounted to $4 \mathrm{~ns}$. The pulse was further compressed to the shortest duration of $3 \mathrm{~ns}$ for the output powers higher than $1 \mathrm{~W}$. At such pump powers, the pulse duration remained nearly unchanged. The fraction of the background in the total emission was greater. At $5 \mathrm{~W}$ of the average output power the spectrum was represented by a broad structured band (Fig. 5). The area under 


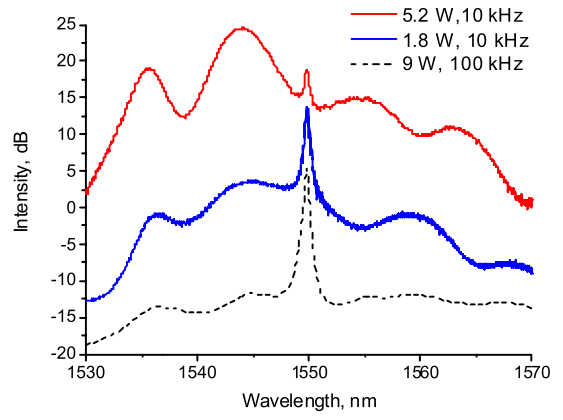

Fig. 5. Emission spectra at $10 \mathrm{kHz}$ and $100 \mathrm{kHz}$ repetition rate.

the narrow line corresponds to the seed laser and amounts to $\sim 1 \%$ from the total spectrally integrated emission.

\section{DISCUSSION}

In this study, we investigated the FBG-stabilized seed laser diode as an alternative to DFB laser diodes for Er-doped fiber amplifiers. FBG stabilization allows perfect lock of the laser wavelength. Although the laser line is significantly broader than a typical linewidth of a DFB laser diode, it is narrow enough for the atmospheric transmission window at $1.5 \mu \mathrm{m}$, and from practical point of view, the narrow linewidth of $0.4 \mathrm{~nm}$ allows the operation in between the intensive absorption lines of the atmosphere after a precise choose of the central wavelength of the FBG-stabilized seed laser diode. The advantage of the FBG-stabilized diode is its high output power. Thus, its average power amounted to 0.5 and $0.4 \mathrm{~W}$ at 10 and $100 \mathrm{kHz}$ repetition rate, respectively. At considerably higher repetition rates beneficial for high acquisition rate systems, the average power of such diodes can be increased up to 1-watt level. However, the high output power of the diode is obtained at the expense of the longer pulse durations. Therefore, the most important advantage of this laser is the essential compression of the pulses propagating in the fiber amplifier. At least 33-fold compression has been demonstrated in our experiment. In such system with the external pulse compression, higher average power of the seed laser is allowed that relaxes requirements to the amplifier. In addition, long pulses allow simpler electronic control. The compression phenomenon originates from the predominant amplification of the forward front of the pulse if the pulse energy becomes comparable or higher than the saturation energy. The amplification of the forward part of the pulse leads to the depletion of the gain for its tail because some ions are falling down from the excited state to the ground state when radiation interacts with matter. If the pulse energy is high enough, all the stored energy comes to the pulse beginning and the amplification of its tail becomes negligible. It is expressed in steeping of the pulse front and its overall shortening. This phenomenon is gain-dependent. Thus, pulses with $10 \mathrm{kHz}$ repetition rate have been better compressed in the $1^{\text {st }}$ stage amplifier as compared to the pulses with $100 \mathrm{kHz}$ repetition rate, Fig. 2. The compression was more efficient because the optical gain accumulated during 10 times longer period between the pulses apparently was higher.
Nearly exponential pulse compression is an efficient tool for obtaining short intensive pulses. The 4-kW level peak power corresponding to the narrow spectral component at the wavelength of $1550 \mathrm{~nm}$ is reasonably high for the practical applications, i.e. remote sensing and micromachining. The observed spectral background currently attributed to ASE can limit the performance of the system. The detailed study of its origin requiring time-resolved spectral measurements, as well as the implementation of the ASE suppression techniques for this system will be performed elsewhere. However, in time domain the background between pulses was negligible (below detection threshold), therefore its appearance in spectra should not affect the detection in ranging applications. At the present stage, we have demonstrated multi-kilowatt output from a simple system, which can be directly applicable in practice being comparable by pulse duration and peak power to the modern commercial systems. This useful technique is promising for LIDARs development as well, as for compression of the optical pulses down to sub-nanosecond durations.

\section{CONClusion}

In this letter we report the Er-doped laser system based on conventional commercially available fibers and operating at the wavelength of $1550 \mathrm{~nm}$ with the average power of up to $10 \mathrm{~W}$. The estimated pulse peak power for the narrow 1550-nm spectral component of $0.4 \mathrm{~nm}$ FWHM exceeded $4 \mathrm{~kW}$ level. A low-power laser diode operating at the repetition rate of $10-100 \mathrm{kHz}$ was used as a seed laser for the twostage fiber amplifier. The first stage amplifier consisted of the core-pumped Er-doped fiber whereas the $2^{\text {nd }}$-stage amplifier was represented by the Er/Yb-doped clad-pumped fiber. The initial pulse duration of $\sim 100$ ns was strongly reduced down to $3.5 \mathrm{~ns}$ as a result of the $34-\mathrm{dB}$ total amplification revealing a powerful nonlinear optical technique for an intense short pulse production. Further investigation of the pulse compression mechanisms will contribute to the development of novel practical, robust, reliable and highly intense pulsed laser devices for numerous applications, e. g. LIDAR applications.

\section{REFERENCES}

[1] C. Cuadrado-Laborde, P. Pérez-Millán, M. V. Andrés, A. Díez, J. L. Cruz, and Y. O. Barmenkov, " Transform-limited pulses generated by an actively $Q$-switched distributed fiber laser," Opt. Lett., vol. 33, no. 22, pp. 2590-2592, Nov. 2008.

[2] S. Adachi and Y. Koyamada, "Analysis and design of Q-switched erbium-doped fiber lasers and their application to OTDR," J. Lightw. Technol., vol. 20, no. 8, pp. 1506-1511, Aug. 2002.

[3] J. Yun et al., "High-peak-power, single-mode, nanosecond pulsed, allfiber laser for high resolution 3D imaging LIDAR system," Chin. Opt. Lett., vol. 10, no. 12, p. 121402, Dec. 2012.

[4] V. Philippov et al., "High-energy in-fiber pulse amplification for coherent LIDAR applications," Opt. Lett., vol. 29, no. 22, pp. 2590-2592, Nov. 2004.

[5] X. Zhang et al., "Eye-safe single-frequency single-mode polarized allfiber pulsed laser with peak power of 361 W," Appl. Opt., vol. 53, no. 11, pp. 2465-2469, Apr. 2014.

[6] Y. Feng, J. Zhou, S. Du, and W. Chen, "All-fiber pulsed laser transmitter for space-based 3D imaging LIDAR," in Proc. CLEO, Pasific Rim, Shanghai, China, Aug. 2009, pp. 1-2.

[7] Y. Liu, J. Liu, and W. Chen, "Eye-safe, single-frequency pulsed allfiber laser for Doppler wind lidar," Chin. Opt. Lett., vol. 9, no. 9, pp. 090604-1-090604-4, Jul. 2011. 
[8] P. Wan, J. Liu, L.-M. Yang, and F. Amzajerdian, "Low repetition rate high energy $1.5 \mu \mathrm{m}$ fiber laser," Opt. Exp., vol. 19, no. 19, pp. 18067-18071, Sep. 2011.

[9] C. Gao, S. Zhu, W. Zhao, Z. Cao, and Z. Yang, "Eye-safe, high-energy, single-mode all-fiber laser with widely tunable repetition rate," Chin. Opt. Lett., vol. 7, no. 7, pp. 611-613, Jul. 2009.

[10] G. Canat, J.-C. Mollier, J.-P. Bouzinac, Y. Aubry, G. Loas, and Y. Jaouën, "100 $\mu \mathrm{J}$ generation using a narrow linewidth $\mathrm{Er}^{+3}-\mathrm{Yb}^{+3}$ doped fiber MOPA and its modeling," in Proc. QELS, Baltimore, MD, USA, May 2005, paper JWB67.

[11] F. Zhang et al., "Impacts of seed power on amplification performance in pulsed double-clad fiber amplifier," Chin. Opt. Lett., vol. 6, no. 1, pp. 19-21, Jan. 2008.

[12] C. E. Dilley, M. A. Stephen, and M. P. Savage-Leuchs, "High SBS-threshold, narrowband, erbium co-doped with ytterbium fiber amplifier pulses frequency-doubled to $770 \mathrm{~nm}, "$ Opt. Exp., vol. 15, pp. 14389-14395, Oct. 2007.

[13] S. Desmoulins and F. D. Teodoro, "High-gain Er-doped fiber amplifier generating eye-safe MW peak-power, mJ-energy pulses," Opt. Exp., vol. 16, no. 4, pp. 2431-2437, Feb. 2008.
[14] D. Taverner, D. J. Richardson, L. Dong, J. E. Caplen, K. Williams, and R. V. Penty, " $158-\mu \mathrm{J}$ pulses from a single-transverse-mode, largemode-area erbium-doped fiber amplifier," Opt. Lett., vol. 22, no. 6, pp. 378-380, Mar. 1997.

[15] B. Desthieux, R. I. Laming, and D. N. Payne, "111kW $(0.5 \mathrm{~mJ})$ pulse amplification at $1.5 \mu \mathrm{m}$ using a gated cascade of three erbiumdoped fiber amplifiers," Appl. Phys. Lett., vol. 63, no. 5, pp. 586-588, May 1993.

[16] A. S. Kurkov, Y. E. Sadovnikova, A. V. Marakulin, and E. M. Sholokhov, "All fiber Er-Tm Q-switched laser," Laser Phys. Lett., vol. 7, no. 11, pp. 795-797, Oct. 2010.

[17] V. V. Dvoyrin, "Pulsed fiber laser with cross-modulation of laser cavities," in Proc. CLEO, San Jose, CA, USA, May 2012, pp. 1-2.

[18] A. G. Okhrimchuk et al.,"Waveguide-saturable absorber fabricated by femtosecond pulses in YAG:Cr4+ crystal for Q-switched operation of Yb-fiber laser," Opt. Lett., vol. 34, no. 24, pp. 3881-3883, Dec. 2009.

[19] A. M. Kowalevicz, Jr., and F. Bucholtz, "Beam divergence from an SMF-28 optical fiber," Naval Res. Lab, Washington, DC, USA, Tech. Rep. NRL/MR/5650-06-8996, Oct. 2006. 